\title{
Effect of Vitamin B Administration on Urine of Mothers - with Negative Arakawa's Reaction.
}

\author{
r4Ist Report of the Petoxidase Reaction.
}

(88th Human Milk Study.)

$\mathrm{By}$

Haruka Umemura.

(梅 村 晴 雷)

(From the Department of Pediatries, Faculty of Medicine, Tohoku Imperial University, Sendai.

Director: Prof. A. Sato.)

In preceding papers ${ }^{1) 2}$ (Cf. 136th Report and 137th Report of the Peroxidase Reaction) I reported of the relation between Arakawa's reaction ${ }^{\dagger}$ and chlorine content of lactating mothers, and of the abnormal retention of chlorine in the body of Arakawa-negative mothers. Muray a ma $\mathrm{a}^{3)}$ found out for the first time that the amount of urine of Arakawa-negative mothers is essentially smaller than that of Arakawa-positive mothers. This assertion has been confirmed by Morika wa $\mathbf{a}^{\ddagger}$ and myself. In the present paper I want to report of the influence of vitamin B upon the chlorine content, the amount and the specific gravity of the urine of mothers with negative A rakaw a's reaction.

Paper read at the General Meeting of the Japanese Pediatric Society held in Kumamoto, April 1939.

* $\mathrm{B}$ means $\mathrm{B}_{1}$ throughout this article.

$\uparrow$ Arakawampsitive may be used in two different senses. One of these is Arakawa-positive in a biochemical sense. A sample of human milk is said to have become Arakawa-positive, when it becomes blue on the addition of Arakawa's reagent. There it means that the sample is not negative to Arakawa's reaction. Arakawapositive in a clinical sense: A sample of human milk is clinically Arakawa-positive only when it shows such a reaction as or in one minute of the addition of A rakawa's reagent. Another sample of human milk may be Arakawa-positive in the first described sense, but yet clinically negative.

1) H. Um e m n ra, Tohoku J. Exp. Med., 1940, 39, 103.

2) H. Um e mu ra, Tohoku J. Exp. Med., 1940, 39, 116.

3) H. Mur a y ma, To be published in this Journal.

$\div$ T. Morikaw a, Not published. 


\section{Materials and Method.}

Materials: Urine voided during 24 hours in the case of lactating mothers, who had, according to what they asserted, no menses, no pregnancy, or no renal diseases during the present experiment.

Vitamin B was injected every 24 hours, and then the urine collector was removed for another urine collector. The amount and specific gravity was noted and then the chlorine content was determined; protein reaction was tried.

Method of Investigation: I applied the blood chlorine method of Rusznyak ${ }^{4}$ to human urine. 1) Protein was tested with $20 \%$ sulfosalicylic acid. Arakawa's reaction was tested within 3 hours of milk obtaining (Cf. 52nd Report of the Peroxidase Reaction) ${ }^{5}$; milk was always obtained before nursing.

\section{Report of Cases.}

Case 1 (Cf. Table 1 and Table 15).

In this case 0.25 mgrms. of vitamin B were injected subcutaneously as often as 19 times, making the total amount of 4.75 mgrms. A rakawa's reaction showed a tendency to become more and more positive after the vitamin administration. The chlorine content of urine was on an a verage $1.03 \mathrm{~g} / \mathrm{dl}$ * before the vitamin administration, but, after this, $0.98 \mathrm{~g} / \mathrm{dl}$ on an average, showing thus a reduction of about $5 \%$. The total urine contained on an average $12.6 \mathrm{grms}$. of chlorin before the vitamin administration, but, after it, 15.4 grms. on an average, showing thus an increase of $22 \%$. In two instances the total chlorin was 11.8 and 12.4 grms. respectively (in contrast to 12.6 grms. before the administration) after the vitamin administration, but such an instance with a reduction of the total chlorin occurred only twice.

The amount of the urine was 1227 c.c. on an average before the vitamin administration. After this, it always showed an increase and was 1574 c.c. on an average, showing an increase of $28 \%$. Only once it was 1130 c.c., smaller than that before the vitamin administration, but this was an exceptional instance. The specific gravity was 1.024 on an average before the vitamin injection, but it was 1.020 after it on an average, showing thus a reduction of $0.4 \%$.

4) St. Rusznyak, Biochem. Z., 1921, 114, 23.

5) Y. Ug a, Tohoku J. Exp. Med., 1934, 24, 208.

* g/dl means grms. per decilitre. 
TABLE 1.

Daily variation of A rakawa's reaction and urine of lactating mother.

Case 1. K. Yamamoto, male, aged 2 months.

Diagnosis: Healthy. Age of mother, 26 years.

\begin{tabular}{|c|c|c|c|c|c|c|c|c|c|c|}
\hline \multirow{2}{*}{ ب } & \multirow{2}{*}{ 煦 } & \multicolumn{2}{|c|}{ Arakawa's reaction } & \multicolumn{2}{|c|}{$\begin{array}{l}\text { Chlorine } \\
\text { content } \\
\text { of urine }\end{array}$} & \multirow{2}{*}{ 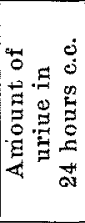 } & \multirow{2}{*}{ 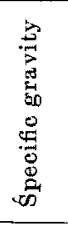 } & \multirow{2}{*}{ 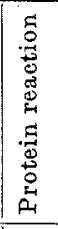 } & \multirow{2}{*}{ 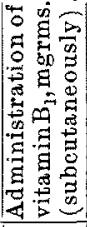 } & \multirow{2}{*}{ 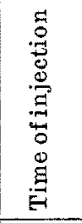 } \\
\hline & & $\begin{array}{l}\text { Right } \\
0^{\prime} 1^{\prime} 2^{\prime} 3^{\prime} 4^{\prime} 5^{\prime}\end{array}$ & $\begin{array}{c}\text { Left } \\
0^{\prime} 1^{\prime} 2^{\prime} 3^{\prime} 4^{\prime} 5^{\prime}\end{array}$ & 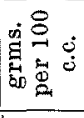 & 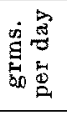 & & & & & \\
\hline 1 & 18. IV & --- \pm \pm \pm & ----- & 0.89 & 12.4 & 1890 & 1025 & $(-)$ & $(-)$ & \\
\hline $\mathbf{2}$ & 19. $n$ & $-1 \pm++1$ & $-\cdots \pm \pm \pm \pm$ & 1.12 & 13.3 & 1190 & 1025 & $(1)$ & $(-)$ & \\
\hline $\mathbf{3}$ & 20. & $+-t+t$ & -- \pm \pm++ & 1.09 & 12.0 & 1100 & 1021 & 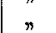 & $n$ & \\
\hline 4 & 22. & $--\cdots \pm \pm$ & $\cdots-- \pm \pm \pm$ & 1.12 & 16.8 & 1500 & 1016 & $\pi$ & 0.25 & 10 p.m \\
\hline 5 & 23. $n$ & --- \pm \pm & ---- \pm \pm & 0.96 & 17.6 & 1830 & 1015 & $n$ & $"$ & $"$ \\
\hline 6 & 24. $n$ & $-\cdots \pm \pm+$ & ---- \pm+ & 0.82 & 15.2 & 1850 & 1017 & $n$ & s & $"$ \\
\hline 7 & 25. $\pi$ & $-m-m-m$ & $\cdots$ & 0.89 & 16.2 & 1820 & 1018 & $"$ & $n$ & $n$ \\
\hline 8 & 26. & $-- \pm \pm+t+$ & $---m \pm$ & 0.96 & 17.6 & 1830 & 1018 & $\pi$ & $n$ & $n$ \\
\hline 9 & 27. & $-\cdots \pm \pm$ & -- \pm \pm \pm & 1.00 & 18.2 & 1820 & 1021 & $n$ & r & $n$ \\
\hline 10 & 28. $n$ & $- \pm++H+$ & $-\cdots \pm \pm+4$ & 1.00 & 15.1 & 1510 & 1018 & 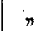 & $n$ & " \\
\hline 11 & 29. n & $- \pm+H H+t$ & $-m \pm \pm++$ & 0.83 & 14.1 & 1700 & 1020 & $n$ & $n$ & $n$ \\
\hline 12 & $30 . \pi$ & $- \pm+H H+H$ & $-\cdots- \pm \pm+\cdots$ & 1.07 & 13.7 & 1280 & 1024 & $n$ & $n$ & $r$ \\
\hline 13 & 1. V & $-4+t+4+4+4$ & $- \pm+4+4+H$ & 1.07 & 14.9 & 1390 & 1024 & $"$ & $"$ & $n$ \\
\hline 14 & 2. $n$ & $-++4+4+H+H$ & $- \pm+4++4$ & 1.07 & 16.9 & 1580 & 1020 & $n$ & $n$ & $"$ \\
\hline 15 & 3. & $\pm++4+4+4$ & $-++H+H+4$ & 0.96 & 16.1 & 1680 & 1021 & $n$ & $\eta$ & $n$ \\
\hline 16 & 4. $n$ & $- \pm \pm+H++$ & $- \pm \pm++t$ & 1.00 & 14.2 & 1420 & 1025 & $"$ & $n$ & $n$ \\
\hline 17 & 5. & $- \pm+\frac{1}{1}+$ & $- \pm+t+t$ & 1.00 & 18.1 & 1810 & 1017 & $n$ & $\eta$ & $\%$ \\
\hline 18 & 6. & $--ー \pm \pm \pm$ & $- \pm+t+H$ & 1.12 & 12.6 & 1130 & 1024 & \# & 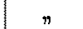 & $n$ \\
\hline 19 & 7. $\pi$ & -- \pm \pm++ & $----ー \pm$ & 0.89 & 12.4 & 1390 & 1020 & " & $n$ & $\eta$ \\
\hline 20 & 8. & $---\cdots \pm$ & $---++H$ & 0.92 & 11.8 & 1280 & 1018 & $\Rightarrow$ & 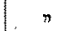 & $n$ \\
\hline 21 & 9. & $- \pm t+H+t$ & $-\cdots \pm++$ & 0.92 & 16.6 & 1800 & 1020 & $n$ & & $n$ \\
\hline 22 & 10. & $- \pm+H+H+H$ & ーー士士士士 & 1.07 & 13.8 & .1290 & 1026 & $n$ & $n$ & $\#$ \\
\hline
\end{tabular}

Case 2 (Cf. Table 2 and Table 15).

In this case 0.25 mgrms. of vitamin $B$ were injected subcutaneously as often as 9 times, making the total amount of 2.25 mgrms. A ra kaw a's reaction showed a tendency to become more and more positive after the vitamin administration. The chlorine content of urine was on an average $0.65 \mathrm{~g} / \mathrm{dl}$ before the vitamin administration, but, after this, $0.73 \mathrm{~g} / \mathrm{dl}$ on an average, showing thus an increase of about $12 \%$. The total urine contained on an average $5.7 \mathrm{grms}$. of chlorin before the vitamin administration, but, after it, 7.6 grms. on an average, showing thus an increase of $33 \%$. After the vitamin administration, in three instances it was 5.0, 5.1 and 5.0 grms. respectively, smaller than that before the vitamin administration.

The amount of the urine was on an average 875 c.c. before the vitamin administration. After this, it always showed an increase (ex- 
cept for two instances in which it was 850 and 750 c.c. respectively) and the amount was $1028 \mathrm{c.c}$. on an average, showing thus an increase of $17 \%$. The specific gravity was on an average 1.015 before the vitamin administration, but it was 1.019 on an average after it, showing an increase of $0.4 \%$.

TABLE 2.

Daily variation of A ralc aw a's reaction and urine of lactating mother.

Case 2. O. Ishihata, male, aged 2 months. Diagnosis: Healthy. Age of mother, 24 years.

\begin{tabular}{|c|c|c|c|c|c|c|c|c|c|c|}
\hline \multirow{2}{*}{ 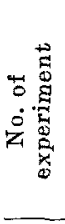 } & \multirow{2}{*}{ 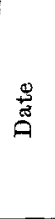 } & \multicolumn{2}{|c|}{ Arakawa's rection } & \multicolumn{2}{|c|}{$\begin{array}{l}\text { Chlorine } \\
\text { content } \\
\text { of urine }\end{array}$} & \multirow{2}{*}{ 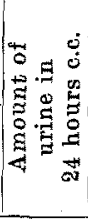 } & \multirow{2}{*}{ 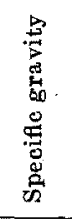 } & \multirow{2}{*}{ 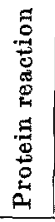 } & \multirow{2}{*}{ 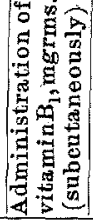 } & \multirow{2}{*}{ 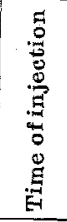 } \\
\hline & & $\begin{array}{c}\text { Right } \\
0^{\prime} 1^{\prime} 2^{\prime} 3^{\prime} 4^{\prime} 5^{\prime}\end{array}$ & $\begin{array}{c}\text { Left } \\
0^{\prime} 1^{\prime} 2^{\prime} 3^{\prime} 4^{\prime} 5^{\prime}\end{array}$ & 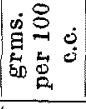 & 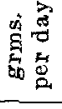 & & & & & \\
\hline 1 & 4. $\mathrm{V}$ & & $\pm t+t+$ & 0.56 & 5.3 & 950 & 1012 & $(-)$ & $(-)$ & \\
\hline 2 & 5. $n$ & \pm \pm+ & $+H+H+H$ & 0.75 & 6.0 & 800 & 1018 & 2, & & \\
\hline 3 & 6. $n$ & $\pm+++H+H$ & $\pm++H+H+H$ & 0.87 & 10.9 & 1250 & 1018 & $n$ & 0.25 & $10 \mathrm{p} . \mathrm{m}$ \\
\hline 4 & 7. & $+4+4$ & \pm++1 & 0.82 & 12.3 & 1500 & 1016 & n & $n$ & $n$ \\
\hline 5 & 8. & $t+t+t$ & $-t+t+4$ & 0.50 & 5.0 & 1000 & 1018 & $n$ & $n$. & $n$ \\
\hline 6 & 9. & $\begin{array}{l}- \pm \pm \\
- \pm \pm+\end{array}$ & $- \pm+4 H+1$ & 0.75 & 6.7 & 900 & 1017 & $\Rightarrow$ & 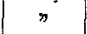 & " \\
\hline $\begin{array}{l}7 \\
8 \\
9\end{array}$ & $\begin{array}{l}10 . \\
11 . \\
12 .\end{array}$ & - \pm \pm+ & $\pm++4+4$ & 0.92 & 7.8 & 850 & 1022 & $"$ & & \\
\hline 10 & 13. $"$ & $-\cdots-- \pm$ & ---- \pm \pm & 0.54 & 5.1 & 950 & 2024 & $( \pm)$ & 0.25 & $10 p \cdot x$ \\
\hline 11 & 14. & $\cdots- \pm \pm \pm$ & - \pm \pm \pm+1 & 0.67 & 5.0 & 750 & 1018 & $( \pm)$ & & \\
\hline 12 & 15. & $-\cdots \pm \pm++$ & $-m \pm \pm \pm$ & 0.71 & 7.4 & 105 & 101 & & & \\
\hline 13 & 16. $n$ & $- \pm+H+H$ & $-1 \pm \pm \pm 1$ & 0.81 & 8.1 & 1000 & 1021 & $(-\infty)$ & & \\
\hline
\end{tabular}

Case 3 (Cf. Table 3 and Table 15).

In this case vitamin $B$ was injected subcutaneously in the amount of $1 \mathrm{mgrm}$. at one time, and in the amount of $0.25 \mathrm{mgrms}$, at the 2 other times, making the total amount of $1.5 \mathrm{mgrms}$. A rakawa's reaction showed a tendency to become more and more positive after the vitamin administration. The chlorine content of urine was on an average 0.68 $\mathrm{g} / \mathrm{dl}$ before the vitamin administration, but, after this, $0.88 \mathrm{~g} / \mathrm{dl}$ on an average, showing thus an increase of $29 \%$. The total urine contained on an average 5.6 grms. of chlorin before the vitamin administration, but, after it, 15.3 grms. on an average, showing thus an increase of $173 \%$. After the vitamin administration, the chlorine content of total urine showed in all the instances an increase.

The amount of the urine was 867 c.c. on an average before the vitamin administration, but subsequently it always showed an increase, and was 1744 c.c. on an average, showing thus an increase of $101 \%$. 
The specific gravity was 1,021 on an average before the vitamin injection, but after it, it was 1.019 on an average, showing a reduction of $0.2 \%$.

\section{TABLE 3.}

Daily variation of A rakawa's reaction and urine of lactating mothers.

Case 3. K. Gonoue, female, aged 19 months. Diagnosis:

Dyspepsia. Age of mother, 26 years.

\begin{tabular}{|c|c|c|c|c|c|c|c|c|c|c|}
\hline \multirow{2}{*}{ 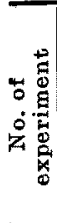 } & \multirow{2}{*}{ 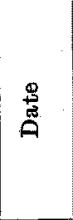 } & \multicolumn{2}{|c|}{ Arak awa's reaction } & \multicolumn{2}{|c|}{$\begin{array}{l}\text { Chlorine } \\
\text { content } \\
\text { of urine }\end{array}$} & \multirow{2}{*}{ 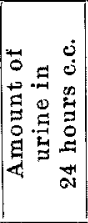 } & \multirow{2}{*}{ 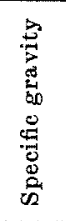 } & \multirow{2}{*}{ 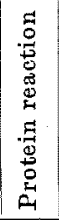 } & \multirow{2}{*}{ 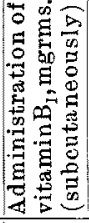 } & \multirow{2}{*}{ 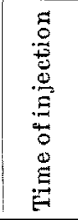 } \\
\hline & & $\begin{array}{c}\text { Right } \\
0^{\prime} 1^{\prime} 2^{\prime} 3^{\prime} 4^{\prime} 5^{\prime}\end{array}$ & $\begin{array}{l}\text { Left } \\
0^{\prime} 1^{\prime} 2^{\prime} 3^{\prime} 4^{\prime} 5^{\prime}\end{array}$ & 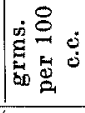 & 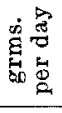 & & & & & \\
\hline 1 & 25. V & $-5--\ldots$ & $-\ldots-\cdots$ & 0.65 & 8.4 & 1300 & 1018 & $(-)$ & $(-)$ & $(-)$ \\
\hline 2 & 26. $\pi$ & --- \pm \pm & ---- \pm & 0.85 & 4.2 & 500 & 1026 & $n$ & & 8 \\
\hline 3 & 27. $\pi$ & $-\cdots-- \pm$ & $-\cdots- \pm$ & 0.53 & 4.2 & 800 & 1020 & $n$ & & $\pi$ \\
\hline 4 & 28. $n$ & $-\cdots \pm+$ & $- \pm+t+t$ & 0.50 & 8.0 & 1600 & 1016 & $n$ & 1.0 & $7 \mathrm{p}, \mathrm{m}$. \\
\hline 5 & $2 . I V$ & $-2 \pm \pm++$ & $\pm++4+4+4$ & 0.58 & 8.7 & 1500 & 1022 & $\pi$ & $(-)$ & $(-)$ \\
\hline 6 & 3. & 土tHHHH & $- \pm \pm+H++$ & 1.02 & 14.8 & 1450 & 1023 & $\pi$ & $n$ & $\eta$ \\
\hline 7 & 4. $n$ & $- \pm \pm+++t$ & $- \pm t++t+1$ & 1.01 & 14.1 & 1400 & 1021 & $n$ & 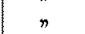 & $\eta$ \\
\hline 8 & 5. & - \pm \pm \pm & $\pm++H+H$ & 0.84 & 19.7 & 2350 & 1018 & $n$ & & \\
\hline 9 & 6. $n$ & -- \pm \pm & $- \pm \pm+t+$ & 1.10 & 15.4 & 1400 & 1022 & $\pi$ & $\pi$ & \\
\hline 10 & 7. $n$ & - \pm \pm+++ & $- \pm++H+H$ & 0.79 & 15.0 & 1900 & 1016 & n & & \\
\hline 11 & 8. $n$ & $-= \pm++H$ & $-- \pm++H$ & 1.07 & 20.3 & 1900 & 1018 & $n$ & 0.25 & 10 p.m. \\
\hline 12 & & $--- \pm+H$ & ---- \pm & 1.01 & 22.2 & 2200 & 1019 & $\pi$ & $n$ & $"$ \\
\hline
\end{tabular}

Case 4 (Cf. Table 4 and Table 15).

In this case vitamin $B$ was injected subcutaneously in the total amount of $29 \mathrm{mgrms}$. A raka w a's reaction showed a tendency to become positive after the injection, though it was later restored to the reaction before it. The chlorine content of urine was on an average $1.02 \mathrm{~g} / \mathrm{dl}$ before the vitamin administration, but after this $0.69 \mathrm{~g} / \mathrm{dl}$ on an average, showing thus a reduction of $32 \%$. The total urine contained 5.1 grms. of chlorin before the vitamin administration, but after it, 13.5 grms. on an average, showing thus a very remarkable increase of $165 \%$. Following the vitamin administration the chlorine content of total urine always showed an increase.

The amount of the urine was 500 c.c. before the vitamin administration. Now after this, it always showed an increase and was 1973 c.c. on an average, showing a very remarkable increase of $295 \%$. The specific gravity was 1.032 before the vitamin injection, but it was 1.016 on an average after it, showing thus a reduction of $1.6 \%$. 


\section{TABLe 4. \\ Daily variation of Arakaw a's reaetion and urine of lactating mother.}

Case 4. E. Eguchi, male, aged 2 months. Diagnosis:

Lues congenita. Age of mother, 39 years.

\begin{tabular}{|c|c|c|c|c|c|c|c|c|c|c|}
\hline \multirow{2}{*}{ 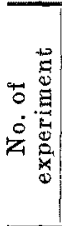 } & \multirow{2}{*}{ 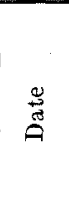 } & \multicolumn{2}{|c|}{ Arakawa's reaction } & \multicolumn{2}{|c|}{$\begin{array}{l}\text { Chlorine } \\
\text { content } \\
\text { of urine }\end{array}$} & \multirow{2}{*}{ 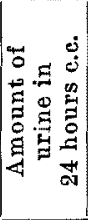 } & \multirow{2}{*}{ 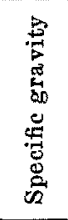 } & \multirow{2}{*}{ 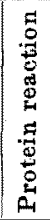 } & \multirow{2}{*}{ 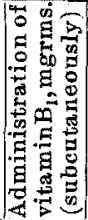 } & \multirow{2}{*}{ 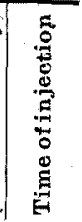 } \\
\hline & & $\begin{array}{c}\text { Right } \\
0^{\prime} I^{\prime} 2^{\prime} 3^{\prime} 4^{\prime} 5^{\prime}\end{array}$ & $\begin{array}{c}\text { Left } \\
0^{\prime} 1^{\prime} 2^{\prime} 3^{\prime} 4^{\prime} 5^{\prime}\end{array}$ & 苞总 & 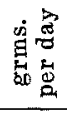 & & & & & \\
\hline 1 & I0. V & $--\cdots--$ & ------ & 1.02 & 5.1 & 500 & 1032 & $(-)$ & $(-)$ & \\
\hline 2 & 11. & $----\cdots$ & $-\cdots-\cdots$ & & & & & 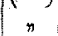 & 0.5 & $10 \mathrm{a}, \mathrm{m}$. \\
\hline 3 & 12." & $---\cdots$ & ------ & 0.78 & 11.7 & 1500 & 1014 & $n$ & 0.5 & $\eta$ \\
\hline 4 & I3. & $--\cdots--$ & $-\cdots----$ & 0.75 & 7.5 & 1000 & 1018 & $n$ & 0.5 & $n$ \\
\hline 5 & 14. $n$ & $-----\cdots$ & $-\cdots---$ & 0.53 & 11.1 & 2100 & 1014 & $n$ & 2.0 & \\
\hline 6 & 15." & $-----\cdots$ & $-\cdots-\cdots$ & 0.74 & 10.0 & 1350 & 1020 & 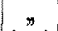 & 3.0 & $5 \mathrm{p.m}$. \\
\hline 7 & $16 *$ & ---- \pm \pm & -ーー上士 & 0.71 & 9.2 & 1300 & 1014 & $( \pm)$ & 2.5 & $10 \mathrm{am}$. \\
\hline 8 & 17. & ---- \pm & ----- & 0.70 & 10.5 & 1500 & 1015 & $(-)$ & 3.0 & $n$ \\
\hline 9 & 18. $n$ & ----- & $-\cdots--\cdots$ & 0.96 & 10.6 & 1100 & 1024 & $( \pm)$ & 5.0 & n \\
\hline 10 & 19." & $-\cdots-\cdots-$ & $\cdots \cdots \pm \pm \pm$ & 0.76 & 13.7 & 1800 & 1014 & $n$ & 5.0 & $n$ \\
\hline 11 & 20. " & $-\cdots \pm \pm \pm$ & - \pm \pm+++ & 0.76 & 14.1 & 1850 & 1016 & $n$ & $(-)$ & \\
\hline 12 & 21." & $---\cdots--$ & --- \pm \pm \pm & 0.63 & 16.4 & 2600 & 1012 & $n$ & 5.0 & $n$ \\
\hline 13 & 22. $n$ & $-\cdots \cdots--$ & $-\cdots-\cdots$ & 0.32 & 7.8 & 2450 & 1024 & $n$ & $(-)$ & \\
\hline 14 & 23. $\pi$ & $-\cdots---$ & $-ー- \pm \pm \pm$ & 0.71 & 14.9 & 2100 & 1016 & $n$ & $n$ & \\
\hline 15 & 24. " & -- \pm \pm \pm \pm & - \pm \pm+++ & 0.69 & 13.8 & 2000 & 1016 & $n$ & $n$ & \\
\hline 16 & 25. & $-ー- \pm \pm \pm$ & $-ー- \pm \pm \pm$ & 0.71 & 11.4 & 1600 & 1022 & $\pi$ & $n$ & \\
\hline .17 & 26. $n$ & $----\cdots$ & $-\cdots \pm \pm++$ & 0.62 & 9.3 & 1500 & 1018 & & $n$ & \\
\hline 18 & 27." & ---- \pm \pm & - \pm \pm \pm+ & 0.50 & 12.5 & 2500 & 1016 & $(-)$ & $n$ & \\
\hline 19 & $28 . \pi$ & $-\cdots- \pm \pm$ & - \pm \pm \pm++ & 0.83 & 18.3 & 1600 & 1010 & $n$ & & ' \\
\hline 20 & $29 . "$ & - \pm \pm+++ & $----\cdots$ & 0.63 & 10.7 & 1700 & 1014 & $n$ & $n$ & \\
\hline 21 & 30. n & $- \pm \pm+1+t$ & - \pm \pm++4 & 0.70 & 15.4 & 2200 & 1012 & $\Rightarrow$ & $\pi$ & \\
\hline 22 & 31. $n$ & - \pm \pm++ & 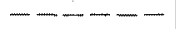 & 0.58 & 9.9 & 1700. & 1018 & $\%$ & " & \\
\hline 23 & $1 . \mathrm{VI}$ & -- \pm \pm \pm+ & -- \pm \pm++ & 0.66 & 16.5 & 2500 & 1014 & $n$ & $n$ & \\
\hline 24 & 2. $n$ & $-\cdots \pm \pm$ & $-+++H+H$ & 0.68 & 11.6 & 1700 & 1016 & y & n & \\
\hline 25 & 3. $n$ & $--\cdots \pm$ & $-\cdots \pm \pm \pm \pm$ & 0.78 & 19,5 & 2500 & 1014 & $n$ & $n$ & \\
\hline 26 & 4." & ----- & $- \pm \pm t++$ & 0.53 & 15.9 & 3000 & 1012 & $\eta$ & $n$ & \\
\hline 27 & 5. $n$ & $-\ldots-\cdots-\cdots$ & $-\cdots-\cdots \pm$ & 0.64 & 16.6 & 2600 & 1016 & $n$ & $n$ & \\
\hline 28 & 6. & ----- & $-\cdots- \pm \pm$ & 0.72 & 20.9 & 2900 & 1013 & & 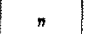 & \\
\hline 29 & 7. $n$ & $-1+t+$ & 一ーー士士土 & 0.78 & 19.5 & 2500 & 1016 & \# & n & \\
\hline 30 & 8. " & $-\ldots-\cdots$ & $-\cdots-\cdots$ & 0.81 & 21.1 & 2600 & 1018 & $n$ & $"$ & \\
\hline 31 & 9. & ---- \pm & $--\cdots--$ & 0.72 & 13.7 & 1900 & 1015 & $n$ & $n$ & \\
\hline 32 & 10. & $-m-\cdots$ & $----\cdots$ & 0.82 & 13.1 & 1600 & 1019 & $n$ & n & \\
\hline 33 & 11., & $-\ldots-\cdots--$ & $--\cdots- \pm$ & 0.81 & 15.4 & 1900 & 1024 & $"$ & $n$ & \\
\hline
\end{tabular}

Case 5 (Cf. Table 5 and Table 15).

In this case 0.5 mgrms. of vitamin B were injected subcutaneously 3 times, making the total amount of 1.5 mgrms. A rakawa's reaction showed a tendency to become more and more positive after the vitamin administration. The chlorine content of urine was $0.90 \mathrm{~g} / \mathrm{dl}$ before the vitamin administration, but after this $0.83 \mathrm{~g} / \mathrm{dl}$ on an average, showing thus a reduction of about $8 \%$. The total urine contained 
12.6 grms. of chlorin before the vitamin administration, but, after it, $15.5 \mathrm{grms}$. on an average, showing thus an increase of $23 \%$. There was one instance in which the total chlorin was 12.3 grms. (in contrast to 12.6 grms. before the vitamin administration) after it, but such an instance with a reduction occurred only once.

The amount of the urine was 1400 c.c. before the vitamin administration. Later, it always showed an increase and 1864 c.c. on an average, showing a rather remarkable increase of $33 \%$. The specific gravity was 1.024 on an average before the vitamin injection, but after it 1.018 on an average, showing a reduction of $0.6 \%$.

\section{TABle 5.}

Daily variation of A rakawa's reaction and urine of lactating mother.

Case 5. S. Yamada, male, aged 2 months. Diagnosis :

Dyspepsia. Age of mother, 23 years.

\begin{tabular}{|c|c|c|c|c|c|c|c|c|c|c|}
\hline \multirow{2}{*}{ 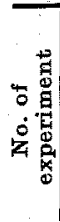 } & \multirow{2}{*}{$\stackrel{9}{9}$} & \multicolumn{2}{|c|}{ Arakawa's reaction } & \multicolumn{2}{|c|}{$\begin{array}{l}\text { Chlorine } \\
\text { content } \\
\text { of urine }\end{array}$} & \multirow{2}{*}{ 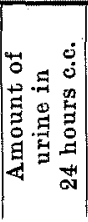 } & \multirow{2}{*}{ 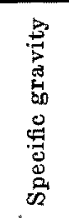 } & \multirow{2}{*}{ 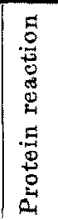 } & \multirow{2}{*}{ 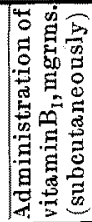 } & \multirow{2}{*}{ 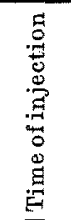 } \\
\hline & & $\begin{array}{c}\text { Right } \\
0^{\prime} 1^{\prime} 2^{\prime} 3^{\prime} 4^{\prime} 5^{\prime}\end{array}$ & $\begin{array}{c}\text { Left } \\
0^{\prime} I^{\prime} 2^{\prime} 3^{\prime} 4^{\prime} 5^{\prime}\end{array}$ & 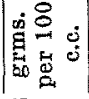 & 总苞 & & & & & \\
\hline $\begin{array}{l}1 \\
2 \\
3 \\
4 \\
5 \\
5\end{array}$ & $\begin{array}{ll}1 . & \text { VII } \\
2 . & 7 \\
3 . & 7 \\
4 . & 7 \\
5 . & 7 \\
6 . & 7\end{array}$ & $\begin{array}{l}-- \pm \pm+ \\
--- \pm++ \\
--- \pm \pm \pm \\
-- \pm+++ \\
-- \pm+++ \\
- \pm+H+H+\end{array}$ & $\begin{array}{l}--- \pm++ \\
---\frac{ \pm}{ \pm} \\
- \pm++H+ \\
-- \pm \pm++ \\
- \pm \pm \pm++ \\
- \pm \pm+ \pm H\end{array}$ & $\begin{array}{l}0.90 \\
0.91 \\
0.89 \\
0.92 \\
0.71 \\
0.72\end{array}$ & $\begin{array}{l}12.6 \\
17.3 \\
16.9 \\
17.7 \\
13.5 \\
12.2\end{array}$ & $\begin{array}{l}1400^{\circ} \\
1900 \\
1900 \\
1920 \\
1900 \\
1700\end{array}$ & $\begin{array}{l}1024 \\
1018 \\
1016 \\
1028 \\
1016 \\
1016\end{array}$ & $\begin{array}{l}(-) \\
" \\
\Rightarrow \\
" \\
\Rightarrow\end{array}$ & $\begin{array}{l}(-) \\
0.5 \\
(-) \\
0.5 \\
(-) \\
0.5\end{array}$ & $9 \mathrm{a}, \mathrm{m}$. \\
\hline
\end{tabular}

Case 6 (Cf. Table 6 and Table 15).

In this case 0.5 mgrms. of vitamin $B$ were injected subcutaneously as often as 11 times, making the total amount of $5.5 \mathrm{mgrms}$. A rakawa's reaction became positive after the vitamin administration. The chlorine content of urine was $1.18 \mathrm{~g} / \mathrm{dl}$ ' on an average, but after. this the average was $0.95 \mathrm{~g} / \mathrm{dl}$, showing thus a reduction of $19 \%$. The total urine contained 5.8 grms. of chlorin on an average before the vitamin administration, but, after it, an average of 8.0 grms., showing thus a remarkable increase of $38 \%$. After the administration, in only one instance did the total chlorin show the same value as the average value ( 5.8 grms.) before the vitamin administration.

The amount of urine was 500 c.c. before the administration, but after this 859 c.c. on an average, showing thus a very remarkable in- 
crease of $72 \%$. In one instance only the amount showed the same average value after the vitamin administration as before it. The specific gravity was 1.020 on an average before the administration, and 1.018 on an average after it, showing thus a reduction of $0.2 \%$.

TABLE 6.

Daily variation of A rakaw's reaction and urine of lactating mother.

Case 6. Y. Sibasaki, male, aged 10 months. Diagnosis : Infantile beriberi. Age of mother, 24 years.

\begin{tabular}{|c|c|c|c|c|c|c|c|c|c|c|}
\hline \multirow{2}{*}{ 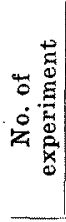 } & \multirow{2}{*}{$\begin{array}{l}\stackrel{0}{+3} \\
\stackrel{2}{2}\end{array}$} & \multicolumn{2}{|c|}{ Arakawa's reaction } & \multicolumn{2}{|c|}{$\begin{array}{l}\text { Chlorine } \\
\text { content } \\
\text { of urine }\end{array}$} & \multirow{2}{*}{ 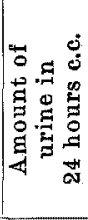 } & \multirow{2}{*}{ 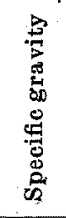 } & \multirow{2}{*}{ 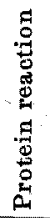 } & \multirow{2}{*}{ 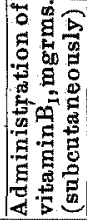 } & \multirow{2}{*}{ 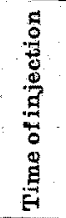 } \\
\hline & & $\begin{array}{c}\text { Right } \\
0^{\prime} 1^{\prime} 2^{\prime} 3^{\prime} 4^{\prime} 5^{\prime}\end{array}$ & $\begin{array}{c}\text { Left } \\
0^{\prime} 1^{\prime} 2^{\prime} 3^{\prime} 4^{\prime} 5^{\prime}\end{array}$ & 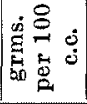 & 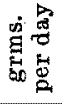 & & & & & \\
\hline 1 & 3. VII & $---1 \pm$ & $-\ldots-\ldots$ & 1.28 & 5.8 & 450 & 1022 & $(-)$ & $(-)$ & \\
\hline 2 & & $-\ldots- \pm \pm$ & $-\cdots \pm \pm$ & 1.25 & 5.7 & 450 & 1023 & 18 & & \\
\hline 3 & & -- \pm \pm \pm & -- \pm \pm \pm & 1.00 & 6.0 & 600 & 1015 & 7 & & \\
\hline 4 & & -- \pm \pm \pm & $-\cdots \pm \pm \pm$ & 1.21 & 8.5 & 700 & 1023 & $m$ & 0.5 & 5 p.m. \\
\hline 5 & & $- \pm+H+H$ & $-D \pm \pm \pm$ & 1.31 & 6.5 & 500 & 1024 & 》 & $\pi$ & $n$ \\
\hline 6 & & -- \pm \pm++ & $- \pm+++t+$ & 0.95 & 12.8 & 1300 & 1016 & m & \% & $n$ \\
\hline 7 & & $- \pm+H+H$ & $- \pm \pm t+H$ & 0.85 & 6.8 & 800 & 1020 & $n$ & 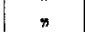 & $n$ \\
\hline 8 & 10. & - \pm \pm \pm \pm \pm & $- \pm++H+t$ & 0.83 & 5.8 & 700 & 1018 & ” & $n$ & $n$. \\
\hline 9 & 11. & $-++4+4$ & $\pm \bar{H}+H+H H$ & 1.03 & 9.8 & 950 & 1018 & 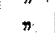 & 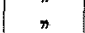 & $n$ \\
\hline 10 & 12. & $-+H+H+4$ & $\pm H+\# \# \#$ & 0.71 & 6.0 & 850 & 1016 & $n$ & $\Rightarrow$ & $\pi$ \\
\hline 11 & 13. & $t+t+t+H+H$ & $\pm++H+H$ & 0.71 & 7.1 & 1000 & 1015 & n & n & \\
\hline 12 & 14. " & $\pm+H+H H$ & +HHHHH & 1.20 & 10.2 & 850 & 1018 & $n$ & & \\
\hline 13 & 15. & $\pm H+H+H$ & 十HHHHH & 0.75 & 7.5 & 1000 & 1014 & $n$ & $n$ & \\
\hline 14 & 16. $n$ & $\pm H+H+H$ & 十世邢州世井 & 0.89 & 7.1 & 800 & 1018 & & $n$ & \\
\hline
\end{tabular}

Case 7 (Cf. Table 7 and Table 15).

In this case vitamin $B$ was injected subcutaneously in the amount of $0.25 \mathrm{mgrms}$. for 3 times and in the amount of $0.5 \mathrm{mgrms}$. on 4 occasions, making the total amount of 2.75 mgrms. Arakawa's reaction became positive after the vitamin administration. The chlorine content of urine was $0.67 \mathrm{~g} / \mathrm{dl}$ on an average before the administration, but, after this, $0.90 \mathrm{~g} / \mathrm{dl}$ on an average, showing thus a remarkable increase of $34 \%$. The total urine contained 14.4 grms. of chlorine before the administration. Now after this, it always showed an increase and was 17.8 grms., showing a rather remarkable increase of $24 \%$.

The amount of the urine was 2150 c.c. before the vitamin administration, and after this 2000 c.c., showing a decrease of $7 \%$. The specific gravity was 1.018 on an average before the vitamin administration, the subsequent average value being 1.019 , thus representing an increase of $0.1 \%$. 
Table 7.

Daily variation of A rak awa's reaetion and urine of lactating mother.

Case 7. H. Numanami, male, aged 2 months. Diagnosis : Infantile beriberi. Age of mother, 28 years.

\begin{tabular}{|c|c|c|c|c|c|c|c|c|c|c|}
\hline \multirow{2}{*}{ 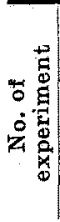 } & \multirow{2}{*}{ 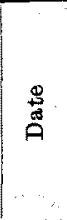 } & \multicolumn{2}{|c|}{ Arakawa's reaction } & \multicolumn{2}{|c|}{$\begin{array}{l}\text { Chlorine } \\
\text { content } \\
\text { of urine }\end{array}$} & \multirow{2}{*}{ 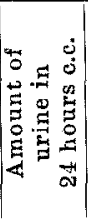 } & \multirow{2}{*}{ 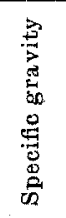 } & \multirow{2}{*}{ 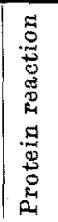 } & \multirow{2}{*}{ 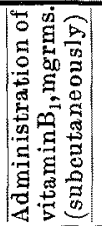 } & \multirow{2}{*}{ 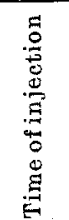 } \\
\hline & & $\begin{array}{c}\text { Right } \\
0^{\prime} 1^{\prime} 2^{\prime} 3^{\prime} 4^{\prime} 5^{\prime}\end{array}$ & $\begin{array}{l}\text { Left } \\
0^{\prime} I^{\prime} 2^{\prime} 3^{\prime} 4^{\prime} 5^{\prime}\end{array}$ & 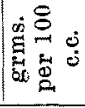 & 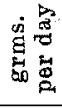 & & & & & \\
\hline $\mathbf{I}$ & 4. VII & $- \pm \pm+H+H$ & -++ H龷H & 0.63 & 13.2 & 2100 & 1018 & $(-)$ & $(-)$ & \\
\hline 2 & & $- \pm+H+H+$ & - \pm \pm \pm+ & 0.71 & 15.6 & 2200 & 1018 & $\pi$ & & \\
\hline 3 & & $- \pm \pm+1+$ & $- \pm \pm++H$ & 0.95 & 16.1 & 1700 & 1018 & $n$ & 0.25 & $1 \mathrm{p} . \mathrm{n}$ \\
\hline 4 & & - \pm \pm \pm++ & - \pm \pm++4 & 0.96 & 18.2 & 1900 & 1020 & * & 0.25 & n \\
\hline 5 & & - $\pm++t+m m$ & $- \pm+t$ & 0.8 & 18.0 & 2200 & 1018 & $\pi$ & 0.25 & " \\
\hline 6 & & $- \pm++4+4$ & $\pm+H+H+4$ & 0.76 & 17.5 & 2300 & 1018 & n & 0.5 & $n$ \\
\hline 7 & 10. $n$ & -十H+H井册 & $-H+H+H+H$ & 0.89 & 17.8 & 2000 & 1018 & n & n & $n$ \\
\hline 8 & 11. & 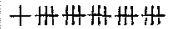 & $+H+H H H+H$ & 0.9 & 19.1 & 2100 & 1018 & $n$ & , & $n$ \\
\hline 9 & 12. & $+H+H+H+H+H$ & 十H+H+H+H & 0.99 & 17.8 & 1800 & 1021 & $\Rightarrow$ & & m \\
\hline
\end{tabular}

Case 8 (Cf. Table 8 and Table 15).

In this case $0.5 \mathrm{mgrms}$. of vitamin $\mathrm{B}$ were injected subcutaneously as often as 6 times, making the total amount of 3.0 mgrms. A ra$\mathrm{k}$ aw a's reaction showed no change in spite of the vitamin administra-

\section{TABle 8.}

Daily variation of Arakawa's reaction and urine of lactating mother.

Case 8. T. Yahagi, male, aged 2 months. Diagnosis: Osteomyelitisiuetica. Age of mother, 24 years.

\begin{tabular}{|c|c|c|c|c|c|c|c|c|c|c|}
\hline \multirow{2}{*}{ 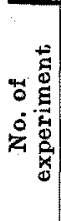 } & \multirow{2}{*}{ 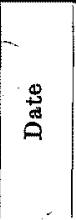 } & \multicolumn{2}{|c|}{ Arakawa's reaction } & \multicolumn{2}{|c|}{$\begin{array}{l}\text { Chlorine } \\
\text { content } \\
\text { of urine }\end{array}$} & \multirow{2}{*}{ 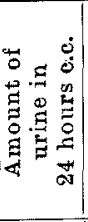 } & \multirow{2}{*}{ 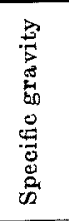 } & \multirow{2}{*}{ 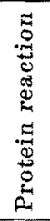 } & \multirow{2}{*}{ 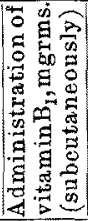 } & \multirow{2}{*}{ 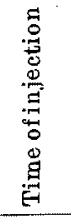 } \\
\hline & & $\begin{array}{c}\text { Right } \\
0^{\prime} 1^{\prime} 2^{\prime} 3^{\prime} 4^{\prime} 5^{\prime}\end{array}$ & $\begin{array}{c}\text { Left } \\
0^{\prime} 1^{\prime} 2^{\prime} 3^{\prime} 4^{\prime} 5^{\prime}\end{array}$ & 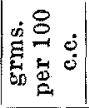 & 总密 & & & & & \\
\hline 1 & $11 . \mathrm{IX}$ & $\ldots \ldots \ldots$ & & 0.77 & 11.5 & 1500 & 1022 & . & $6(-)$ & \\
\hline 2 & I2. & ----- & $\pm \pm t++t$ & 0.8 & 12.7 & 1550 & 1020 & 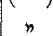 & $"$ & \\
\hline 3 & 13. 7 & ----- \pm & \pm \pm++++ & 0.79 & 12.2 & 1550 & 1021 & $n$ & $n$ & \\
\hline 4 & 14. & $--\cdots++$ & $\pm++H$ & 0.98 & 14.7 & 1500 & 1020 & $"$ & & \\
\hline 5 & 15. $\pi$ & --- \pm \pm & $++H$ & 0.8 & 13.6 & 1550 & 1020 & $n$ & 0.5 & $1 \mathrm{p.m}$. \\
\hline 6 & 16. $"$ & $\cdots-\cdots \pm$ & $\pm \pm++4+4$ & 0.62 & 14.3 & 2300 & 1020 & $n$ & $n$ & $n$ \\
\hline 7 & 17. & $----\cdots$ & \pm++++4 & 0.86 & 20.6 & 2400 & 1023 & $\$$ & $n$ & $"$ \\
\hline 8 & 18. & -- \pm \pm \pm & $\pm+H+H+H$ & 0.7 & 18.9 & 2450 & 1017 & $n$ & 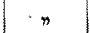 & $n$ \\
\hline 9 & 19. & ---- \pm & $\pm \pm++++H$ & 0.70 & 16.4 & 2350 & 1019 & $n$ & $n$ & $n$ \\
\hline 10 & & ----- & $\pm \pm+H+H$ & 0.65 & 15.3 & 2350 & 1018 & & $n$ & * \\
\hline
\end{tabular}


tion. The chlorine content of urine was on an average $0.84 \mathrm{~g} / \mathrm{dl}$, but after this $0.75 \mathrm{~g} / \mathrm{dl}$ on an average, showing thus a reduction of $11 \%$. The total urine contained an average of 12.8 grms. of chlorine. After this it always showed an increase, the average figure bêing 16.5 grms. thus showing a rather remarkable increase of $20 \%$.

The amount of urine was on an average 1525 c.c. before the vitamin administration, but after this 2233 c.c. on an average, showing teus a remarkable increase of $46 \%$. After the administration the amount of urine always increased. The specific gravity was 1.021 on an average before the vitamin injection, but after it, it was 1.020 on an average, showing thus a decrease of $0.1 \%$.

Case 9 (Cf. Table 9 and Table 15).

In this case 0.5 mgrms. of vitamin $B$ were injected subcutaneously as often as 9 times, making the total amount of $4.5 \mathrm{mgrms}$. A rakawa's reaction became positive after the vitamin administration. The chlorine content of urine was on an average $0.59 \mathrm{~g} / \mathrm{dl}$ before the administration, but after it $0.65 \mathrm{~g} / \mathrm{dl}$ on an average, showing an increase of $10 \%$. The total urine contained on an average 9.2 grms. of chlorine before the vitamin administration, and after it $10.3 \mathrm{grms}$. on an aver-

TABLE 9.

Daily variation of A rakawa's reaction and urine of lactating mother.

Case 9. T. Takahashi, fomale, aged 10 months. Diagnosis: Anemia. Age of mother, 25 years.

\begin{tabular}{|c|c|c|c|c|c|c|c|c|c|c|}
\hline \multirow{2}{*}{ 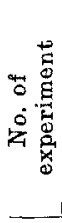 } & \multirow{2}{*}{ 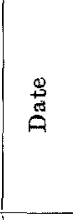 } & \multicolumn{2}{|c|}{ Arakaw a's reaction } & \multicolumn{2}{|c|}{$\begin{array}{l}\text { Chlorine } \\
\text { content } \\
\text { of urine }\end{array}$} & \multirow{2}{*}{ 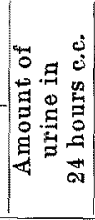 } & \multirow{2}{*}{ 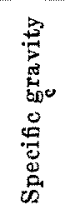 } & \multirow{2}{*}{ 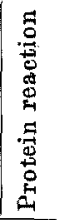 } & \multirow{2}{*}{ 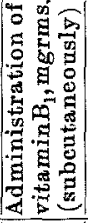 } & \multirow{2}{*}{ 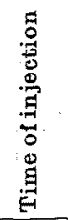 } \\
\hline & & $\begin{array}{c}\text { Right } \\
0^{\prime} 1^{\prime} 2^{\prime} 3^{\prime} 4^{\prime} 5^{\prime}\end{array}$ & $\begin{array}{c}\text { Left } \\
0^{\prime} 1^{\prime} 2^{\prime} 3^{\prime} 4^{\prime} 5^{\prime}\end{array}$ & 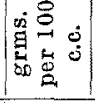 & 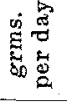 & & & & & \\
\hline 1 & 7. X & $\pm t+1$ & 一土士十+H情 & 0.56 & 9.2 & 1650 & 1015 & $(-)$ & $(-)$ & \\
\hline 2 & 28." & $\pm+4+4+H+4$ & \pm++ & 0.64 & 9.9 & 155 & 10 & t & & \\
\hline 3 & 29. & 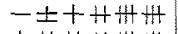 & $+H+H+4$ & 0.54 & 10.5 & 1950 & 1016 & & $n$ & \\
\hline 4 & $30 .=$ & $+4+4+4$ & - +HH姍H & 0.64 & 8.3 & 1300 & 1018 & & & \\
\hline 5 & 31. " & & - $+H_{3}+4$ & 0.59 & 8.3 & 1400 & 1015 & & & \\
\hline 6 & 1.XI & +4 & \pm++ & 0.75 & 12.4 & 1650 & 1016 & " & 0.5 & $5 \mathrm{p.m}$ \\
\hline 7 & 2." & \pm \pm & $-+t$ & 0.65 & 9.1 & 1400 & 1017 & " & $\pi$ & \\
\hline 8 & 3. $"$ & 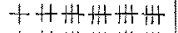 & - \pm+ & 0.65 & 10.1 & 1560 & 1018 & & , & \\
\hline 9 & & 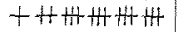 & $-- \pm+H$ & 0.65 & 8.4 & 1300 & 1015 & $\eta$ & & \\
\hline 10 & & $+H+H+H+H+H$ & -士十+H+H & 0.59 & 8.5 & 1450 & 1016 & 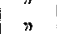 & & \\
\hline 11 & & $+\mathrm{H} H+H+H$ & $-\frac{1}{+}+H+H$ & 0.57 & 10.5 & 1850 & 1015 & $\eta$ & & 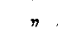 \\
\hline 12 & & 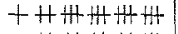 & $-+H+H H$ & 0.65 & 10.1 & 1550 & 1015 & $n$. & & \\
\hline 13 & 8. & $\pm H+H+H+H$ & $\pm H+H+H$ & 0.65 & 11.4 & 1750 & 1017 & & & $n$ \\
\hline 14 & & $\pm H+4+4+4$ & $\pm H+H+H$ & 0.70 & 11.9 & 1700 & 1018 & " & & \\
\hline
\end{tabular}


age, showing thus an increase of $12 \%$. After the vitamin administration; in three instances, the chlorine content of the total urine was 9.1, 8.4 and 8.6 grms. respectively, smaller than the average value before the vitamin administration.

The amount of the urine was on an average 1570 c.c. before the vitamin administration and after it 1579 c.c. on an average, showing thus a reduction of $1 \%$. The specific gravity showed no change resulting from the vitamin injection.

Case 10 (Cf. Table 10 and Table 15).

In this case 0.5 mgrms. of vitamin B were injected subcutaneously as often as 5 times, making the total amount of $2.5 \mathrm{mgrms}$. A rakawa's reaction was already positive before the injection, but it became more strongly positive after it. The chlorine content of urine was on an average $0.86 \mathrm{~g} / \mathrm{dl}$ before the vitamin administration, but, after this, $0.79 \mathrm{~g} / \mathrm{dl}$ on an average, showing thus a decrease of $8 \%$. The total urine contained on an average $9.19 \mathrm{grms}$. of chlorin before the vitamin administration, but, after this, 9.24 grms. on an average, showing an increase of $0.5 \%$.

The amount of the urine was 1082 c.c. on an average before the vitamin administration; afterwards this was 1174 c.c. on an average, showing an increase of $8 \%$. The average specific gravity was 1.027

\section{TAble 10.}

Daily variation of A r a kawa's reaction and urine of lactating mother.

Case 10. K. Kato, male, aged 11 months. Diagnosis:

Pneumonia. Age of mother, 26 years.

\begin{tabular}{|c|c|c|c|c|c|c|c|c|c|c|}
\hline \multirow{2}{*}{ 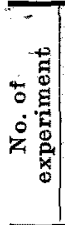 } & \multirow{2}{*}{ 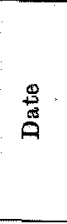 } & \multicolumn{2}{|c|}{ Arakawa's reaction } & \multicolumn{2}{|c|}{$\begin{array}{l}\text { Chlorine } \\
\text { content } \\
\text { of urine }\end{array}$} & \multirow{2}{*}{ 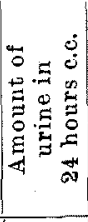 } & \multirow{2}{*}{ 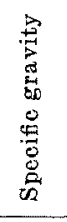 } & \multirow{2}{*}{ 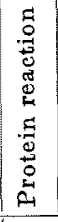 } & \multirow{2}{*}{ 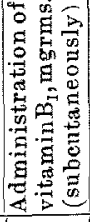 } & \multirow{2}{*}{ 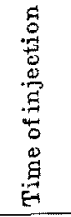 } \\
\hline & & $\begin{array}{c}\text { Right } \\
0^{\prime} 1^{\prime} 2^{\prime} 3^{\prime} 4^{\prime} 5^{\prime}\end{array}$ & $\begin{array}{c}\text { Left } \\
0^{\prime} 1^{\prime} 2^{\prime} 3^{\prime} 4^{\prime} 5^{\prime}\end{array}$ & 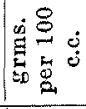 & 总密 & & & & & \\
\hline 1 & 26. $X$ & $+H H H H$ & $-+H+H+H+H$ & 0.67 & 9.4 & 1400 & 1027 & $(-)$ & $(-)$ & \\
\hline 2 & 27. & $\pm+\#+\#+\#$ & $- \pm+H+$ & 0.91 & 9.7 & 1070 & 1029 & $n$ & $n$ & \\
\hline 3 & 28. $\pi$ & $- \pm H+1+H$ & $- \pm H+H+H$ & 0.86 & 10.3 & 1200 & 1022 & $\Rightarrow$ & $\pi$ & \\
\hline 4 & 29. $n$ & -土HHHH & $- \pm+H+H$ & 0.91 & 7.5 & 820 & 1028 & $\Rightarrow$ & 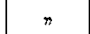 & \\
\hline 5 & 30. n & $+H H+H+H$ & $\pm+H+H H+H$ & 0.94 & 6.6 & 700 & 1029 & 川 & $n$ & \\
\hline 6 & 3I. $\pi$ & 士HHHH+H & $\pm+H+H+H+H$ & 0.89 & 11.6 & 1300 & 1025 & n & $n$ & \\
\hline 7 & $1 . \mathrm{XI}$ & 十廾H\#車舟州 & $\pm+H H H H$ & 0.78 & 11.3 & 1450 & 1027 & $n$ & 0.5 & 1 p.m. \\
\hline 8 & 2. & $-H+4+H 4$ & 二HHHHH & 0.82 & 8.2 & 1000 & 1021 & ᄁ & $n$ & $n$ \\
\hline 9 & 3. & 一井舟州H & - HHWHH & 0.75 & 10.9 & 1450 & 1022 & n & 》 & $\pi$ \\
\hline 10 & 4. \# & 士\#\#\#井H㘫 & $\pm H+H+H H+H$ & 0.82 & 8.2 & 1000 & 1020 & $\pi$ & $n$ & * \\
\hline 11 & 5. $\pi$ & 士H世H州H & 土世H+HHHH & 0.78 & 7.6 & 970 & 1020 & " & $n$ & $\#$ \\
\hline
\end{tabular}


and 1.022 before and after the vitamin injection respectively, which means that there was a reduction of $0.5 \%$.

Case 11 (Cf. Table 11 and Table 15).

In this case $1.0 \mathrm{mgrm}$. of vitamin $B$ was injected subcutaneously 4 times, making the total amount of $4.0 \mathrm{mgrms}$. Arakawa's reaction showed a tendency to become more and more positive after the vitamin administration. The chlorine content of urine was on an average $0.91 \mathrm{~g} / \mathrm{dl}$ before the vitamin administration, but after this $0.98 \mathrm{~g} / \mathrm{dl}$ on an average, showing thus an increase of about $8 \%$. The total urine contained on an average $7.7 \mathrm{grms}$. of chlorin before the vitamin administration, but, after it 9.4 grms. on an average, showing thus a rather remarkable increase of $22 \%$. Following the vitamin administration the chlorine content of the total urine always showed an increase in contrast to $7.7 \mathrm{grms}$, the average before it.

The amount of the urine was 865 c.c. on an average before the vitamin administration, but it always showed an increase afterwards, and was 992 c.c. on an average, showing an increase of $15 \%$. In one instance only it was 700 c.c., smaller than the average before the vitamin administration, but this was an exception. The average specific gravity was 1.025 before the injection, and 1.025 after it, showing a reduction of $0.1 \%$.

Table 11.

Daily variation of A r a t a wa's reaction and urine of lactating mother.

Case 11. R. Sato, male, aged 10 months. Diagnosis:

Pneumonia. Age of mother, 22 years.

\begin{tabular}{|c|c|c|c|c|c|c|c|c|c|c|}
\hline \multirow{2}{*}{ 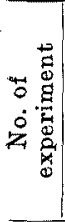 } & \multirow{2}{*}{ 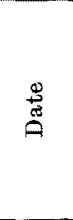 } & \multicolumn{2}{|c|}{ Arakawa's reaction } & \multicolumn{2}{|c|}{$\begin{array}{l}\text { Chlorine } \\
\text { content } \\
\text { of urine }\end{array}$} & \multirow{2}{*}{ 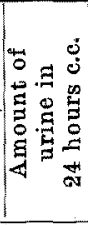 } & \multirow{2}{*}{ 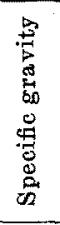 } & \multirow{2}{*}{ 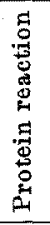 } & \multirow{2}{*}{ 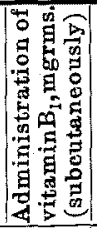 } & \multirow{2}{*}{ 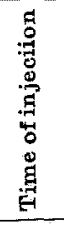 } \\
\hline & & $\begin{array}{c}\text { Right } \\
0^{\prime} 1^{\prime} 2^{\prime} 3^{\prime} 4^{\prime} 5^{\prime}\end{array}$ & $\begin{array}{c}\text { Left } \\
0^{\prime} 1^{\prime} 2^{\prime} 3^{\prime} 4^{\prime} 5^{\prime}\end{array}$ & 足 & 总兽 & & & & & \\
\hline 1 & 15. XI & - \pm \pm & $4+$ & 0.8 & 8.8 & 1100 & & - & $(-)$ & \\
\hline 2 & & -- \pm & +4 & 0. & 4. & 81 & & & & \\
\hline 3 & 18. & -- \pm & $+t+$ & 1.0 & 12.1 & 112 & & & & \\
\hline 4 & 20." & $\cdots \pm \pm$ & $+\mathrm{Tt}$ & 1.1 & 5. & 43 & & & & \\
\hline 5 & 21. " & --1 & +4 & 1.1 & 11.2 & 98 & 10 & & 1.0 & $9 \mathrm{a} . \mathrm{m}$ \\
\hline 6 & 22. " & $-\cdots \pm$ & +4 & 1.1 & 8.2 & 70 & 108 & & $n$ & $n$ \\
\hline 7 & & 143 & $1=1$ & 0.76 & 10.5 & 138 & 10 & $n$ & $n$ & $n$ \\
\hline 8 & & $\pm t+$ & -1 & 0.87 & 7.9 & 910 & 1024 & & $\pi$ & $n$ \\
\hline
\end{tabular}

Case 12 (Cf. Table 12 and Table 15). 
In this case 0.5 mgrms. of vitamin $B$ were injected subcutaneously 3 times, making the total amount of 1.5 mgrms. Arakawa's reaction showed a tendency to become more and more positive after the vitamin administration. The chlorine content of urine was on an average $1.08 \mathrm{~g} / \mathrm{dl}$ before, and $1.03 \mathrm{~g} / \mathrm{dl}$ on an average after the vitamin administration, showing thus a reduction of about $5 \%$. The total urine contained on an average 6.2 grms. of chlorin before the vitamin administration, but, after it, 9.4 grms. on an average, thus showing a remarkable increase of $52 \%$. After vitamin it always showed an increase in contrast to 6.2 grms., the average before the administration.

The amount of urine was 574 c.c. on an average before the vitamin administration, whereas it always showed a very remarkable increase of $70 \%$ afterwards. In only one instance it was 550 c.c., smaller than the average before the vitamin, but this was an exceptional instance. The specific gravity was 1.033 on an average before the vitamin injection, but after it 1.026 was the average amount, thus showing a reduction of $0.7 \%$.

\section{Table 12.}

Daily variation of A rakawa's reaction and urine of lactating mother.

Case 12. M. Yokolawa, male, aged 12 months. Diagnosis: Pneumoni Age of mother, 24 years.

\begin{tabular}{|c|c|c|c|c|c|c|c|c|c|c|}
\hline \multirow{2}{*}{ 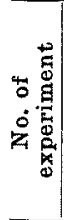 } & \multirow{2}{*}{ 韋 } & \multicolumn{2}{|c|}{ Arakawas reaction } & \multicolumn{2}{|c|}{$\begin{array}{l}\text { Chlorine } \\
\text { content } \\
\text { of urine }\end{array}$} & \multirow{2}{*}{ 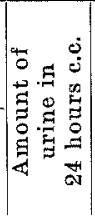 } & \multirow{2}{*}{ 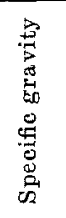 } & \multirow{2}{*}{ 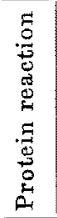 } & \multirow{2}{*}{ 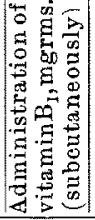 } & \multirow{2}{*}{ 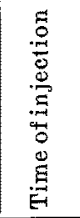 } \\
\hline & & $\begin{array}{c}\text { Right } \\
0^{\prime} 1^{\prime} 2^{\prime} 3^{\prime} 4^{\prime} 5^{\prime}\end{array}$ & $\begin{array}{c}\text { Left } \\
0^{\prime} 1^{\prime} 2^{\prime} 3^{\prime} 4^{\prime} 5^{\prime}\end{array}$ & 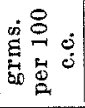 & 离 & & & & & \\
\hline 1 & $16 . \mathrm{XI}$ & $一 \pm \pm \pm \pm \pm$ & $1+1$ & 1.18 & 5.2 & 440 & 1037 & $-)$ & $(-)$ & \\
\hline 2 & 17. & $- \pm+++1+$ & $- \pm+1+1$ & 1.04 & 6.0 & 580 & 1034 & -1 & $n$ & \\
\hline 3 & 18. & -- \pm \pm \pm+ & - \pm++++ & 1.13 & 7.9 & 700 & 1034 & $"$ & $n$ & \\
\hline 4 & 19. $\%$ & $- \pm++1+1$ & $- \pm+t+t$ & 1.11 & 6.7 & 600 & 1034 & \% & 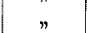 & \\
\hline 5 & 20. & - \pm \pm \pm++ & - \pm \pm+++ & 0.90 & 8.6 & 960 & 1026 & $n$ & $"$ & \\
\hline 6 & 21. & $--\ldots \pm \pm$ & - \pm \pm \pm \pm & 1.09 & 6.1 & 560 & 1083 & $n$ & " & \\
\hline 7 & 22. & - - - - & $- \pm++1+$ & 1.10 & 3.0 & 270 & 1036 & $n$ & $"$ & \\
\hline 8 & 23. & - \pm \pm+++ & - \pm \pm++1 & $\ldots$ & - & 430 & 1035 & $"$ & $"$ & \\
\hline 9 & 24. & 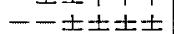 & $-+++++t$ & $\therefore$ &... & 350 & 1035 & * & * & \\
\hline 10 & 25. & $\cdots \pm \pm \pm \pm$ & $-+t+t+H$ & - & - & 850 & 1026 & $n$ & & \\
\hline 11 & 26. & -土土+十+ & $\pm++t++t$ & 1.18 & 6.5 & 550 & 1030 & , & 0.5 & $10 \mathrm{a.m}$. \\
\hline 12 & 27. & - \pm \pm \pm++ & 一土土土t+ & 0.90 & 11.5 & 1280 & 1023 & $n$ & $n$ & $n$ \\
\hline 13 & 28., & ーニーーー士 & - \pm++++ & 0.93 & 10.2 & 1100 & 1024 & $\pi$ & $n$ & $"$ \\
\hline
\end{tabular}

Case 13 (Cf. Table 13 and Table 15).

In this case $1.0 \mathrm{mgrm}$, of vitamin $B$ was injected subcutaneously 
as often as 6 times, making the total amount of $6.0 \mathrm{mgrms}$. A rakawa's reaction showed a tendency to become nore and more positive after the vitamin administration. The chlorine content of urine was on an average $0.92 \mathrm{~g} / \mathrm{dl}$ before the vitamin administration, but after this $0.98 \mathrm{~g} / \mathrm{dl}$ on an average, showing thus an increase of $6 \%$. The total urine contained on an average 7.8 grms. of chlorin before, and 9.6 grms. on an average after the vitamin administration, showing thus a rather remarkable increase of $23 \%$. After the vitamin there was one instance in which the total chlorin was $7.5 \mathrm{grms}$. (in contrast to 7.8 grms., the average before the administration), but such an instance with a reduction occurred only once.

The amount of the urine was 893 c.c. on an average before the vitamin administration, but after it 1000 c.c. on an average, showing an increase of $12 \%$. In only two instances after the vitamin administration it was 700 c.c. and 750 c.c. respectively, smaller than the average before the vitamin administration. The specific gravity was 1.025 on an average before the vitamin injection, but it was 1.026 on an average after it, showing an increase of $0.1 \%$.

\section{TABle 13.}

Daily variation of A r a $k a w a$ 's reaction and urine of lactating mother.

Case 13. I. Yamada, female, aged 10 months. Diagnosis:

Pneumonia. Age of mother, 28 years.

\begin{tabular}{|c|c|c|c|c|c|c|c|c|c|c|}
\hline \multirow{2}{*}{ 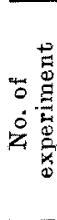 } & \multirow{2}{*}{ 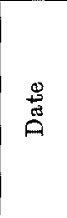 } & \multicolumn{2}{|c|}{ Arakawa's reaction } & \multicolumn{2}{|c|}{$\begin{array}{l}\text { Chlorine } \\
\text { content } \\
\text { of urine }\end{array}$} & \multirow{2}{*}{ 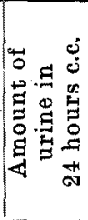 } & \multirow{2}{*}{ 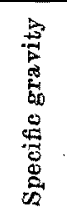 } & \multirow{2}{*}{ 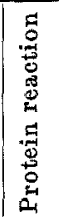 } & \multirow{2}{*}{ 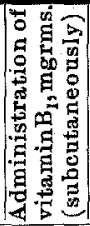 } & \multirow{2}{*}{ 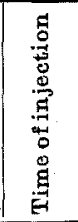 } \\
\hline & & $\begin{array}{l}\text { Right } \\
0^{\prime} 1^{\prime} 2^{\prime} 3^{\prime} 4^{\prime} 5^{\prime}\end{array}$ & $\begin{array}{l}\text { Left } \\
0^{\prime} 1^{\prime} 2^{\prime} 3^{\prime} 4^{\prime} 5^{\prime}\end{array}$ & 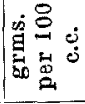 & 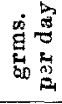 & & & & & \\
\hline 1 & 5. XII & $-\cdots- \pm \pm$ & $--\cdots \pm \pm$ & 0.8 & 7.8 & 970 & 1020 & $(-)$ & $(-)$ & \\
\hline 2 & & --- \pm++ & 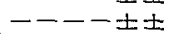 & 1.1 & 6. & 560 & & 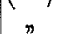 & & \\
\hline 3 & & $- \pm+t+1$ & $-\cdots \pm \pm \pm \pm$ & 0.81 & 9. & 1150 & 108 & $n$ & & \\
\hline 4 & & $---\cdots \pm$ & $-\cdots--$ & 0.80 & 8.8 & 1100 & 102 & $n$ & 1.0 & 10 a.m. \\
\hline 5 & & $--\cdots \pm \pm$ & $--\cdots- \pm$ & 1.0 & 7.5 & 700 & 1022 & $\pi$ & ^ & $n$ \\
\hline 6 & 10. $n$ & $-\cdots-- \pm \pm$ & $-\cdots \pm++$ & 1.1 & 8.4 & 750 & 103 & $\eta$ & $n$ & $n$ \\
\hline 7 & 12." & $1+t+t$ & $\pm+++H+H$ & 1.06 & 10.1 & 950 & 1029 & $n$ & n & $\because$ \\
\hline 8 & 13. $\pi$ & $\pm \pm+H+H$ & $- \pm \pm+t+$ & 0.9 & 12.7 & 1400 & 1022 & $n$ & & \\
\hline 9 & 15.7 & $\pm \pm \pm++H$ & - \pm \pm \pm++ & 0.90 & 9.9 & 1100 & 1020 & 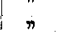 & & \\
\hline
\end{tabular}

Case 14 (Cf. Table 14 and Table 15).

In this case $0.5 \mathrm{mgrm}$. of vitamin B were injected subcutaneously as often as 8 times, making the total amount of $4.0 \mathrm{mgrms}$. A raka- 
wa's reaction showed a tendency to become more and more positive after the vitamin administration. The chlorine content of urine was on an average $1.05 \mathrm{~g} / \mathrm{dl}$ before the vitamin administration, but after this $1.17 \mathrm{~g} / \mathrm{dl}$ on an average, showing thus an increase of $11 \%$. The total urine contained on an average 6.6 grms. of chlorin before the

\section{Table 14.}

Daily variation of Arakawa's reaction and urine of lactating mother.

Case 14. T. Obara, female, aged 7 months. Diagnosis:

Healthy. Age of mother, 32 years.

\begin{tabular}{|c|c|c|c|c|c|c|c|c|c|c|}
\hline \multirow{2}{*}{ 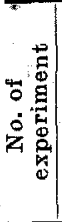 } & \multirow{2}{*}{ 岕 } & \multicolumn{2}{|c|}{ Arakawa's reaction } & \multicolumn{2}{|c|}{$\begin{array}{l}\text { Chlorine } \\
\text { content } \\
\text { of urine }\end{array}$} & \multirow{2}{*}{ 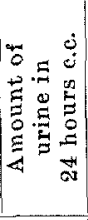 } & \multirow{2}{*}{ 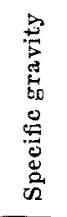 } & \multirow{2}{*}{ 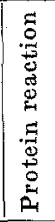 } & \multirow{2}{*}{ 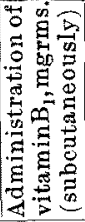 } & \multirow{2}{*}{ 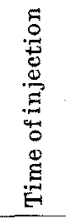 } \\
\hline & & $\begin{array}{c}\text { Right } \\
0^{\prime} 1^{\prime} 2^{\prime} 3^{\prime} 4^{\prime} 5^{\prime}\end{array}$ & $\begin{array}{c}\text { Left } \\
0^{\prime} 1^{\prime} 2^{\prime} 3^{\prime} 4^{\prime} 5^{\prime}\end{array}$ & 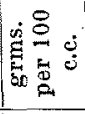 & 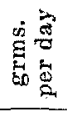 & & & & & \\
\hline 1 & 17. II & & $- \pm \pm+H$ & 1.06 & 10.6 & 1000 & 1032 & - & 1 & \\
\hline 2 & 18. & HH & -- \pm \pm++ & 1.04 & 6.4 & 620 & 1034 & $\pi$ & $\pi$ & \\
\hline 3. & 19.", & $\pm H+H+H+H$ & -- \pm+++ & 1.04 & 8.6 & 350 & 1082 & $n$ & n & \\
\hline 4 & 20. $\pi$ & $\pm+H$ 米册州 & -- \pm++ & 1.09 & 6.0 & 550 & 1034 & 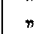 & & 9.30 \\
\hline 5 & 21. & $\pm H+4+4+4$ & --- \pm \pm & 1.07 & 11.2 & 1050 & 1030 & r & 0.5 & a.m. \\
\hline 6 & 23. & $+H+H+H+H$ & $- \pm+H H H$ & 1.15 & 7. & 640 & 1036 & $n$ & $n$ & $n$ \\
\hline 7 & & $+H H H H H$ & $-- \pm+H+4$ & 1.31 & 11.8 & 900 & 1032 & $"$ & \# & $n$ \\
\hline 8 & 25. $n$ & $+H+H+H$ & -- \pm++4 & 1.18 & 5.9 & 500 & 1034 & & $n$ & \\
\hline 9 & & $+H H+H+H$ & $\therefore \pm+H+H$ & 1.26 & 10.6 & 840 & 1034 & 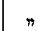 & " & $n$ \\
\hline 10 & 28. & $+H \# \# \# \#$ & $-- \pm+H+H$ & 1.98 & 9.8 & 1000 & 1028 & $r$ & $n$ & $\pi$ \\
\hline
\end{tabular}

vitamin administration, but, after it, 9.4 grms. on an average, showing thus an increase of $42 \%$. There was one instance in which the total chlorin was 5.9 grms. (in contrast to 6.6 grms. before the administration) after the vitamin administration, but such an instance with a reduction occurred only once.

TABLE 15.

Average figures of urine chlorin and urine amount before and after the vitamin administration.

\begin{tabular}{|c|c|c|c|c|c|}
\hline & \multirow{2}{*}{ Cases and number } & \multicolumn{2}{|c|}{$\begin{array}{l}\text { Chlorin in } \\
\text { urine }\end{array}$} & \multirow{2}{*}{$\begin{array}{c}\text { Amount } \\
\text { of } \\
\text { urine }\end{array}$} & \multirow{2}{*}{$\begin{array}{l}\text { Specific } \\
\text { gravity }\end{array}$} \\
\hline & & $\begin{array}{c}\text { grms. } \\
\text { per } 100 \\
\text { c.e. }\end{array}$ & $\begin{array}{c}\text { grms. } \\
\text { per day }\end{array}$ & & \\
\hline Case 1 & $\begin{array}{l}\text { Average before the administration } \\
\text { Average after the administration } \\
\text { Deviation }\end{array}$ & $\begin{array}{c}1.03 \\
0.98 \\
-\quad 5 \%\end{array}$ & $\begin{array}{r}12.6 \\
15.4 \\
+\quad 22 \%\end{array}$ & $\begin{array}{c}1227 \\
1574 \\
+\quad 28 \%\end{array}$ & $\begin{array}{c}1024 \\
1020 \\
-0.4 \%\end{array}$ \\
\hline
\end{tabular}


Table 15. Continued.

\begin{tabular}{|c|c|c|c|c|c|}
\hline & \multirow{2}{*}{ Cases and number } & \multicolumn{2}{|c|}{$\begin{array}{l}\text { Chlorine in } \\
\text { urine }\end{array}$} & \multirow{2}{*}{$\begin{array}{c}\text { Amount } \\
\text { of } \\
\text { urine }\end{array}$} & \multirow{2}{*}{$\begin{array}{l}\text { Specifie } \\
\text { gravity }\end{array}$} \\
\hline & & $\begin{array}{c}\text { grms. } \\
\text { per } 100 \\
\text { e.e. }\end{array}$ & $\begin{array}{l}\text { grms. } \\
\text { per day }\end{array}$ & & \\
\hline Case 2 & $\begin{array}{l}\text { Average before the administration } \\
\text { Average after the administration } \\
\text { Deviation }\end{array}$ & $\begin{array}{c}0.65 \\
0.73 \\
+\quad 12 \%\end{array}$ & $\begin{array}{c}5.7 \\
7.6 \\
+\quad 33 \%\end{array}$ & $\begin{array}{r}875 \\
1028 \\
+\quad 17 \%\end{array}$ & $\begin{array}{c}1015 \\
1019 \\
+0.4 \%\end{array}$ \\
\hline Case 3 & $\begin{array}{l}\text { Average before the administration } \\
\text { Average after the administration } \\
\text { Deviation }\end{array}$ & $\begin{array}{c}0.68 \\
0.88 \\
+\quad 29 \%\end{array}$ & $\begin{array}{r}5.6 \\
15.3 \\
+175 \%\end{array}$ & $\begin{array}{r}867 \\
1744 \\
+101 \%\end{array}$ & $\begin{array}{c}1021 \\
1019 \\
-0.2 \%\end{array}$ \\
\hline Case 4 & $\begin{array}{l}\text { Value before the administration } \\
\text { Average after the administration } \\
\text { Deviation }\end{array}$ & $\begin{array}{c}1.02 \\
0.69 \\
-32 \%\end{array}$ & $\begin{array}{c}5.1 \\
13.5 \\
+165 \%\end{array}$ & $\begin{array}{c}500 \\
1973 \\
+295 \%\end{array}$ & $\begin{array}{c}1032 \\
1016 \\
-1.6 \%\end{array}$ \\
\hline Case 5 & $\begin{array}{l}\text { Value before the administration } \\
\text { Average after the administration } \\
\text { Deviation }\end{array}$ & $\begin{array}{r}0.90 \\
0.83 \\
-\quad 8 \%\end{array}$ & $\mid \begin{array}{c}12.6 \\
15.5 \\
+23 \%\end{array}$ & $\begin{array}{c}1400 \\
1864 \\
+\quad 33 \%\end{array}$ & $\begin{array}{c}1024 \\
1018 \\
-0.6 \%\end{array}$ \\
\hline Case 6 & $\begin{array}{l}\text { Average before the administration } \\
\text { Average after the administration } \\
\text { Deviation }\end{array}$ & $\begin{array}{c}1.18 \\
0.95 \\
-19 \%\end{array}$ & $\begin{array}{l}5.8 \\
8.0 \\
+\quad 38 \% \\
\end{array}$ & $\begin{array}{r}500 \\
859 \\
+\quad 72 \%\end{array}$ & $\begin{array}{l}1020 \\
1018 \\
-0.2 \%\end{array}$ \\
\hline Case & $\begin{array}{l}\text { Average before the administration } \\
\text { Average after the administration } \\
\text { Deviation }\end{array}$ & $\begin{array}{c}0.67 \\
0.90 \\
+\quad 34 \%\end{array}$ & $\begin{array}{r}14.4 \\
17.8 \\
+\quad 24 \%\end{array}$ & $\begin{array}{r}2150 \\
2000 \\
-\quad 7 \%\end{array}$ & $\begin{array}{r}1018 \\
1019 \\
+0.1 \% \\
\end{array}$ \\
\hline Case 8 & $\begin{array}{l}\text { Average before the administration } \\
\text { Average after the administration } \\
\text { Deviation }\end{array}$ & $\begin{array}{c}0.84 \\
0.75 \\
-\quad 11 \%\end{array}$ & $\begin{array}{r}12.8 \\
16.5 \\
+\quad 29 \%\end{array}$ & $\begin{array}{r}1525 \\
2283 \\
+\quad 46 \%\end{array}$ & $\begin{array}{c}1021 \\
1020 \\
-0.1 \%\end{array}$ \\
\hline Case 9 & $\begin{array}{l}\text { Average before the administration } \\
\text { Average after the administration } \\
\text { Deviation }\end{array}$ & $\begin{array}{c}0.59 \\
0.65 \\
+\quad 10 \%\end{array}$ & $\begin{array}{c}9.24 \\
10.3 \\
+12 \% \\
\end{array}$ & $\begin{array}{c}1570 \\
1579 \\
+\quad 1 \%\end{array}$ & $\begin{array}{l}1016 \\
1016 \\
0 \%\end{array}$ \\
\hline Case 10 & $\begin{array}{l}\text { Average before the administration } \\
\text { Average after the administration } \\
\text { Deviation }\end{array}$ & $\begin{array}{c}0.86 \\
0.79 \\
-\quad 8 \%\end{array}$ & $\begin{array}{c}9.19 \\
9.24 \\
+0.5 \%\end{array}$ & $\begin{array}{r}1082 \\
1174 \\
+\quad 8 \%\end{array}$ & $\begin{array}{r}1027 \\
1022 \\
-0.5 \%\end{array}$ \\
\hline $\mathrm{Ca}$ se 11 & $\begin{array}{l}\text { Average before the administration } \\
\text { Average after the administration } \\
\text { Deviation }\end{array}$ & $\begin{array}{r}0.91 \\
0.98 \\
+\quad 8 \% \\
\end{array}$ & $\begin{array}{l}7.7 \\
9.4 \\
+\quad 22 \%\end{array}$ & $\begin{array}{c}865 \\
992 \\
+\quad 15 \%\end{array}$ & $\begin{array}{l}1025 \\
1024 \\
-0.1 \%\end{array}$ \\
\hline Case 12 & $\begin{array}{l}\text { Average before the administration } \\
\text { Average after the administration } \\
\text { Deviation }\end{array}$ & $\begin{array}{c}1.08 \\
1.03 \\
-\quad 5 \%\end{array}$ & $\begin{array}{r}6.2 \\
9.4 \\
+52 \%\end{array}$ & $\begin{array}{r}574 \\
977 \\
+\quad 70 \%\end{array}$ & $\begin{array}{c}1033 \\
1026 \\
-0.7 \%\end{array}$ \\
\hline Case 13 & $\begin{array}{l}\text { Average before the administration } \\
\text { Average after the administration } \\
\text { Deviation }\end{array}$ & $\begin{array}{c}0.92 \\
0.98 \\
+\quad 6 \%\end{array}$ & $\begin{array}{r}7.8 \\
9.6 \\
+23 \% \\
\end{array}$ & $\begin{array}{r}893 \\
1000 \\
+\quad 12 \% \\
\end{array}$ & $\begin{array}{r}1025 \\
1026 \\
+0.1 \% \\
\end{array}$ \\
\hline Case 14 & $\begin{array}{l}\text { Average before the administration } \\
\text { Average after the administration } \\
\text { Deviation }\end{array}$ & $\begin{array}{c}1.06 \\
1.17 \\
+\quad 11 \%\end{array}$ & $\begin{array}{r}6.6 \\
9.4 \\
+\quad 42 \%\end{array}$ & $\begin{array}{c}630 \\
822 \\
+\quad 29 \%\end{array}$ & $\begin{array}{l}1033 \\
1083 \\
0 \%\end{array}$ \\
\hline
\end{tabular}


TABIE 16.

A ralk awa's reaction before the vitamin administration, and the results (shown in deviation) after it.

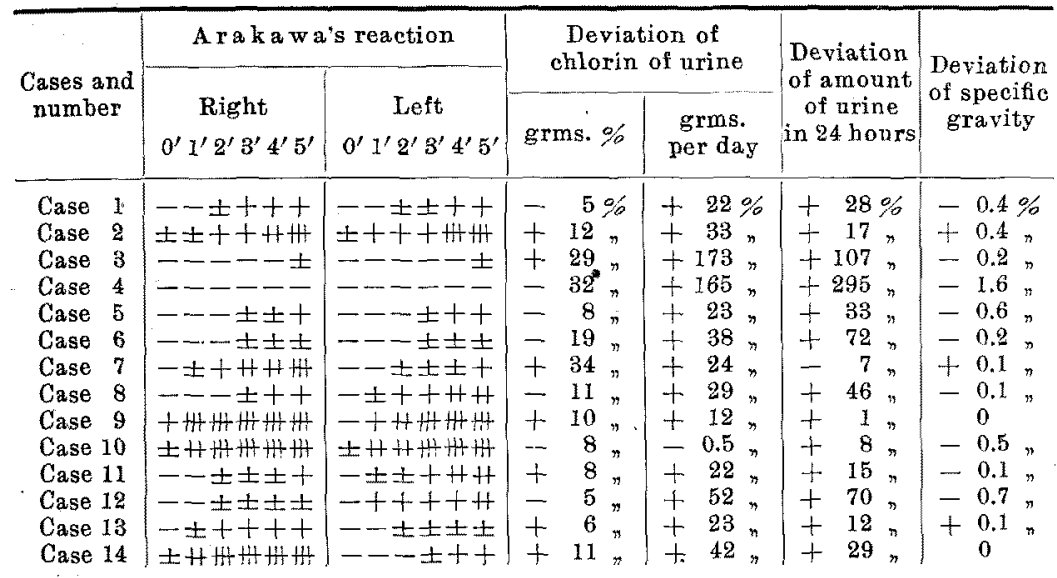

Table 17.

Table 16, very simplified.

\begin{tabular}{l|r|r}
\hline Arakawa's reaction & $\begin{array}{c}\text { Chlorine content of } \\
\text { total urine }\end{array}$ & Amount of total urine \\
\hline (-) Negative & $165 \%-170 \%(170 \%)$ & $107 \%-295 \%(200 \%)$ \\
$( \pm)$ Weakly positive & $22 \%-52 \%(31 \%)$ & $12 \%-72 \%(31 \%)$ \\
$(+)$ Positive & $12 \%-05 \%(6 \%)$ & $1 \%-8 \%(1 \%)$
\end{tabular}

The amount of the urine was 630 c.c. on an average before the vitamin administration, but after this, it always showed an increase, and was 822 c.c. on an average, thus increasing $29 \%$. In only one instance it was 500 c.c., smaller than the average before the vitamin administration, but this was an exceptional instance. The specific gravity was 1.033 on an average before the vitamin injection, and it was 1.033 on an average after it, so no increase came about.

\section{Comment.}

1. Arakawa's reaction.

The reaction in Cases 6.7 and 9 became positive after the vitamin injection. The reaction in Case 10 was already positive before the injection, but became more strongly positive. In Case 4 , the reaction showed a tendency to become positive after the injection, though it 
was later restored to the reaction before it. Case 8 showed no change of A rakawa's reaction in spite of the vitamin injection. These two cases (Cases 4 and 8 ) were syphilitic, and it was no wonder that their Arakawa's reaction did not improve, as has already been shown by Is hii (Cf. 87th Report of the Peroxidase Reaction) ${ }^{6}$ ) and by Yoshino (Cf. 101st Report of same) ${ }^{7)}$ especially.

2. Chlorine content of urine.

The percentage of chlorine content did not, as is shown in Table 16 , show a definite change after the vitamin administration, but the amount of chlorine in the whole day urine increased definitely in all the cases. This increase was especially remarkable in Case 4, in which Arakawa's reaction was very, very weak with (-) $15^{\prime *}$, and in Case 3 with almost as weak a reaction as in Case 4 . And in Case 9 in which Arakawa's reaction was already almost positive, the increase of chlorin after the vitamin injection was not remarkable. As to Case 10 with positive Arakawa's reaction, the increase was the minimum $(0.5 \%)$ or zero. The reason why the increase of the whole day amount of chlorin was the most remarkable in cases with negative Arakawa's reaction and the least remarkable in cases with positive reaction is very simply explained, if the results published in my preceding papers ${ }^{12)}$ are taken into consideration. In Arakawa-negative cases an abnormal retention of chlorin occurs in the body, while in Arakawa-positive mothers little or no retention is seen. So mothers with negative A rakawa's reaction will show an increase of chlorine excretion per day after an administration of vitamin $B$, while no increase, or almost no increase will be seen in spite of the administration in mothers with positive Arakaw a's reaction.

3. The amount of urine.

As is shown in Table 16, the amount of urine showed after the administration of vitamin B an increase in all the cases except for Case 7, in which a slight decrease was seen. The increase was especially remarkable in Cases 3 and 4 with negative Araka w a's reaction; it was $107 \%$ in Cases 3 and 295\% in Case 4 . In Case 9 with almost positive A rakawa's reaction and in Case 10 with positive reaction before the vitamin administration, the increase was only $1 \%$ and $8 \%$ respectively, so that it was negligible or insignificant. This result will demon-

6) M. Is hii, Tohoku J. Exp. Med., 1935, 27, 532.

7) K. Yoshino, Tohokn J. Exp. Med., 1938, 34, 319.

* ( - 15' means "ever negative" after a lapse of 15 minutes of the performance of Arakawa's reaction. 
strate the fact that in mothers with negative Arakawa's reaction, a ( $-\mathrm{n}$ abnormal) retention of water is present, and that in cases with positive A rakawa's reaction such a retention will not occur. Or, an administration of vita min $B$ will produce an augmentation of urine excretion in mothers with negative Arakawa's reaction, while it will not increase the amount of urine in mothers with positive A rakawa's reaction.

4. Specific gravity.

As is shown in Table 16, the administration of vitamin B produced no change of specific gravity in Cases 9 and 14, an increase in Cases 2, 7 and 13, and a decrease in all the other nine cases. In Cases 2 and 7 , the specific gravity was already low (1.015 in Case 2, 1.018 in Case 7) before the vitamin administration. The above result shows that the administration of vitamin $B$ will make the specific gravity of urine smaller in general, except for cases in which it was already low before the administration. From this result, it is interesting to see that the specific gravity is generally higher in the urine of Arakawa-negative mothers than in that of mothers with positive A rakawa's reaction.

5. The colour of urine.

The urine of Arakawa-positive mothers is generally clean, yellowish and transparent, but that of Arakawa-negative ones is mostly unclean, brown and appears more or less turbid. After treating Arakawanegative mothers with vitamin $\mathrm{B}$, the unclean urine gradually becomes as clean yellowish and transparent as that of Arakawa-positive ones.

What has been described will be restated below:-

1. In mothers with negative A rakaw a's reaction, the administration of vitamin $B$ will shift the reaction toward positive, and increase the whole day chlorine excretion and the urine output.

Now, Uehara, So and $\mathrm{Fujinam} \mathrm{i}^{\mathrm{s})}$ made an investigation into chlorin metabolism in beriberi and B-avitaminosis. An administration of vitamin B in avitaminotic cases with edema would, they asserted, result in the excretion $3 \sim 4$ times as much as chlorine in the urine as these patients had taken. $\mathrm{Hasui}{ }^{9}$ ) also found a decrease of urine chlorin and urine output in the stage of exacerebation of beriberi. Omori, Ohashi, Nakanishi, Hara and $\mathrm{Ota}^{10)}$ fed patients slightly affected with beriberi on a B-avitaminotic diet, and found a de-

8) Uehara, So and Fujinami, Tokyo Igakkai Zasshi, 1922, 36, 142.

9) N. Ha sui, Nippon Naika-gakkai Zasshi, 1922, 10, 169.

10) K. Omori, Y. Ohashi, J. Nakanishi, M. Hara and T. Ota, Keio Igaku, $1928,3,197$. 
crease of urine chlorin and urine output in the stage of exacerebation of the disease, and an increase of urine chlorin and urine output in the stage of improvement. Then they experimented on feeding healthy persons on a B-avitaminotic diet and found a decrease of urine chlorin and urine output. Inawashiro and Ichimi1) experimented on feeding 2 healthy persons on a B-avitaminotic diet for a long time and found a remarkable increase of urine output after the vitamin administration. Recently $\mathrm{Masuko}^{12}$ injected subcutaneously into healthy male rabbits of about 2 kilos $50 \sim 10$ c.c. of spelzon, paranutrin or $50 \sim 20$ c.c. of $1 \%$ beriberol pur., and saw no increase of urine output. Hecht and $\mathrm{Wees} \mathrm{e}^{13)}$ reported that diuresis of rabbits in hunger with a peroral administration of 100 grms. of water and with a subcutaneous injection of vitamin $B$ in the amount of $0.1 \mathrm{~g}$. per kilo gram of body became twice as strong as that of the controls without the vitamin injection. Schretzenmayr ${ }^{14)}$ stated in a symposium that though even in very pronounced cases of hydropic form of a deficiency disease the edema is of such a form between myxedema and true edema, and though finger impressions are often of short duration, in milder cases of B-avitaminosis hydropic symptoms are relatively frequent. Catullo Fiorio $0^{15}$ investigated the diuretic effect of the vitamin $B$ recently and obtained the following result. He administered healthy and unhealthy persons 900 c.c. perorally in the morning when they were fasting, and then 2 mgrms. of vitamin B subcutaneously. In this water-experiment he saw an augment of diuresis amounting to $200 \%$ in unhealthy cases, while no diuretic effect of the vitamin was, he stated, seen in healthy cases. Compare this result with my own; almost no diuretic effect of the vitamin in Arakawa-positive mothers, and the striking effect of it in Arakawa-negative cases.

The result of experiments above cited is in agreement with my own result, and will explain that mothers with positive A rakawa's reaction are generally healthy or almost healthy, and that mothers with negative reaction are unhealthy or B-avitaminotic. This will further prove that human milk with positive Arakawa's reaction is generally a "good" milk, and that Arakawa-negative milk is generally a "bad" milk.

11) R. In a w a siro and T. I chimi, Nisshin Igaku, 1928, 17, 1798.

12) T. Masuko, Zika Zassi (Acta Paediatrica Japonica), 1936, 248, 147.

13) G. Hecht and H. We ese, Klin. Wschr., 1937, 12, 414.

14) A. Schretzen mayr, Klin. Wschr., 1937, 50, 1737.

15) Catullo Fiorio, Klin. Wschr., 1938, 38, 1054. 


\section{Summary.}

Human milk negative to Arakawa's reaction is generally Bavitaminotic milk as has has been shown by a number of papers from our Laboratory. Mothers with negative Arakawa's reaction will show an increase of chlorine excretion and urine output per day after an administration of vitamin $B$, while little or no increase will be seen in spite of the administration in mothers with positive Arakawa's reaction.

The reason why the increase of the whole day amount of chlorin was the most remarkable in cases with negative A rakaw a's reaction, and the least remarkable in cases with positive reaction is very simply explained, if the results published in my preceding papers are taken into consideration. In Arakawa-negative cases an abnormal retention of chlorin occurs in the body, while in Arakawa-positive mothers very little retention, if any, is seen. A remarkable increase in urine output after an administration of vitamin $B$ will demonstrate the fact that in mothers with negative A raka wa's reaction a ( $-\mathrm{n}$ abnormal) retention of water occurs.

The result of experiment of other authors above cited is parallel with my own result. It is very probable that, viewed from the effect of vitamin $B$ on urine of mothers with negative A rakawa's reaction, the Arakawa-negative human milk will pass for a B-avitaminotic milk* even to the exclusion of other points of view.

\section{Conclusions.}

1. In mothers with negative Arakawa's reaction, the administration of vitamin $B$ will shift the reaction toward positive, and increase the whole day chlorine excretion and the urine output.

2. In the mothers with positive A rakawa's reaction, the administration will make the positive reaction possibly more intense, and may cause a small increase of the whole day chlorin excretion and the urine output.

3. Specific gravity will generally be made lower except for cases in which it is already low before the administration of vitamin $B$.

4. This will further prove that human milk with positive Arawa's reaction is generally a good milk, and that Arakawa-negative milk is generally a bad milk.

5. The general impression may be expressed in this way:-

Aralcawa-negative mother +vitamin $B=$ Arakawa-positive mother.

* Meaning a milk from a B-avitaminotic body. 